\title{
Occupational Health Nurses' Participation in Health Committee Surveyed in Yamaguchi Prefecture, Japan
}

\author{
Masaiwa INOUE ${ }^{1 *}$, Shinji YAMAMOTO ${ }^{2}$, Hirohiko KAN ${ }^{3}$, \\ Hajime TATEISHI ${ }^{4}$ and Noriaki HARADA ${ }^{1}$
}

\author{
${ }^{1}$ Department of Hygiene, Yamaguchi University School of Medicine, 1-1-1 Minamikogushi, Ube-City, Yamaguchi \\ 755-8505, Japan \\ ${ }^{2}$ Medical Care Center, Nisshin Steel Co., LTD, 4976 Nomuraminami, Shunan-City, Yamaguchi 746-8666, Japan \\ ${ }^{3}$ Health Care \& Support Center, Ube Industries, LTD, 1978-10 Kogushi, Ube-City, Yamaguchi 755-8633, Japan \\ ${ }^{4}$ Department of Medical Care, Teijin Co., LTD, 2-1, Hinode-machi, Iwakuni-City, Yamaguchi 740-8511, Japan
}

Received January 17, 2003 and accepted November 6, 2003

\begin{abstract}
This survey was conducted to know the present conditions of occupational health nurses (OHNs) activity related to health committees (HCs) in Japan. Questionnaires that included items related to duties of the $\mathrm{OH}$ physician (OHP), those of the OHN, and their mutual duties within the $\mathrm{HC}$, were mailed to 41 companies employing OHNs and questionnaires from 18 companies were analyzed. Comparison of the frequency of $\mathrm{OHN}$ attendance at Health Committee Meetings (HCMs) revealed that $33.3 \%$ of OHNs attended the HCs when their companies employed full-time OHPs and $83.3 \%$ attended when their companies employed part-time OHPs. In a question about the OHN's opportunity to deliver a speech, give a report or make a presentation at their HCMs, $16.7 \%$ of OHNs in companies with full-time OHPs and $66.7 \%$ of OHNs in companies with part-time OHPs reported they had such opportunity. In companies with part-time OHPs, $\mathbf{5 0 . 0 \%}$ of the OHNs reported that they were asked for their opinions at the HCMs, but OHNs at companies employing full-time OHPs were not asked for their opinions. It was considered that in the future, OHNs, particularly those working with a part-time OHP, will have an important role in the $\mathrm{HC}$ and developing $\mathrm{OH}$ services for their companies.
\end{abstract}

Key words: Occupational health nurse, Occupational health physician, Health committee meeting, Occupational health service, Questionnaire

\section{Introduction}

It has been almost twelve years since Japan faced serious economic recession and was forced to make radical changes to stabilize the economy. Although various economic countermeasures were attempted, the situation has not been changed; the unemployment rate has increased in recent years, and it is not rare for small and medium-sized companies to declare bankruptcy. Naturally, the wave of recession has eroded not only the economic life of individual citizens but also their mental welfare. More than 30,000 people were

*To whom correspondence should be addressed. killed by themselves every year for 4 continuous years, 19982001. Mental health specialists in Japan speculate that the increase in suicides may be related to economic problems ${ }^{1)}$. It is natural that critical economic changes in a society will create changes in the workplace, with a subsequent demand for high quality occupational health $(\mathrm{OH})$ services for workers, and that the entire $\mathrm{OH}$ system in Japan is likely to undergo scrutiny and redefinition ${ }^{2}$. The role of the $\mathrm{OH}$ nurse (OHN) should receive as much attention as the role of the $\mathrm{OH}$ physician (OHP), because large companies, which are required to employ full-time OHPs, will decrease under the economic reconstruction phenomenon. Research on working conditions for OHNs shows that they need more organized 
support to improve health management in companies ${ }^{3)}$. It has also been proposed in a study on OHNs' role that OHN education programs should be introduced systematically). Although an $\mathrm{OHN}$ has lesser authority on $\mathrm{OH}$ system in companies than an $\mathrm{OHP}$, (s)he is expected to promote $\mathrm{OH}$ system quality. Promotion of $\mathrm{OH}$ service would be helpful to mental health problems in current Japan society. There is a little information on $\mathrm{OHN}$ activities regarding practical tasks in companies; however, this issue needs to be addressed. Therefore, we conducted a survey-based study of one local area in Japan to investigate OHN's activity. In this survey, we focused on a health committee (HC), such committees are required by law in Japan in companies with more than 50 employees, and discussed present conditions pertaining to the OHN's role in the field. Attendance in the health committee meeting (HCM) is not demanded for an OHN while it is recommended for an OHP by the law. However, it is difficult for a part-time OHP to attend the meeting, and then we consider a role of an OHN to attend the HCM. This is the reason to feature up information from a $\mathrm{HC}$ in this study.

\section{Materials and Methods}

Our survey was conducted in Yamaguchi prefecture, which is in the western part of Japan. Questionnaires that included items on OHN working conditions were mailed to 41 companies employing OHNs belonging to the Yamaguchi Occupational Nurse Research Association. In a cover letter asking for participation in the survey, we requested an answer of the question from OHNs of the companies. The cover letter included notes that the confidentiality is maintained and the use of data obtained is confined to the survey. The questionnaires comprised items related to duties of the OHP, those of the OHN, and their mutual duties within the HC. After completion, questionnaires were returned by fax with no sender name. Because the fax address was in an office of Yamaguchi Occupational Physician Association, the person on duty deleted the sender name on the questionnaires if written. We collected questionnaires from 19 companies but included only 18 in our analysis, since one questionnaire from a health examination agency was determined unsuitable.

\section{Results}

Among the 18 companies, OHPs were employed full-time in 6 and part-time in 12. The work frequency for part-time OHPs varied from twice a year to twice a week. We compared OHN attendance in HCMs across companies employing full- time OHPs and those employing part-time OHPs. HCMs were conducted in all 18 companies. Comparison of the frequency of OHP attendance in HCMs revealed that 83.3\% of full-time OHPs and $41.7 \%$ of part-time OHPs attended more than half of the HCMs held, whereas $33.3 \%$ of OHNs attended the HCMs when their companies employed fulltime OHPs and $83.3 \%$ attended when their companies employed part-time OHPs. The rate of assigned roles to OHNs in the HCM was $16.7 \%$ when their companies employed full-time OHPs and $58.3 \%$ when part-time OHPs. In a question about the OHN's opportunity to deliver a speech, give a report or make a presentation at their HCMs, $16.7 \%$ of OHNs in companies with full-time OHPs and $66.7 \%$ of OHNs in companies with part-time OHPs reported that they had such opportunity. In companies with part-time OHPs, $50.0 \%$ of the OHNs reported that they were asked for their opinions at the HCMs; $33.3 \%$ of those OHNs proposed a larger $\mathrm{OHN}$ role in $\mathrm{OH}$ services, but $\mathrm{OHNs}$ at companies employing full-time OHPs were not asked for their opinions and they reported that they did not often offer them. A report on the HCM was routinely sent to OHNs in $66.7 \%$ of companies with full-time OHPs and in $91.7 \%$ of companies with part-time OHPs. Although OHNs in companies with full-time OHPs attended infrequently in duties relating to the $\mathrm{HC}, 41.7 \%$ of OHNs in companies employing part-time OHPs were involved with $\mathrm{HC}$ office tasks, $25.0 \%$ introduced new information on $\mathrm{OH}$ services at the $\mathrm{HCM}, 50.0 \%$ reported information about health examinations, $25.0 \%$ presented health education materials, and $16.7 \%$ conducted HC meetings. Finally, we analyzed the frequency of walk through surveys made by the OHP and the OHN. While $83.3 \%$ of full-time OHPs made walk through surveys in the company more than once a month, only $41.7 \%$ of part-time OHPs did so. $\mathrm{OHN}$ attendance in walk through surveys was $50.0 \%$ when an OHP was employed on full-time basis and $66.7 \%$ when an OHP was employed on part-time basis. P values are shown in Table 1 indicating no significant difference between companies employing full-time OHPs and those employing part-time.

\section{Discussion}

Although there was no significant difference in answers, we found a tendency that the OHN played a more active role in the HCM in companies employing part-time OHPs than in companies employing full-time OHPs. While 33\% of OHNs attended the HCM in companies employing fulltime OHPs, $83 \%$ of OHNs did in those employing part-time OHPs $(\mathrm{p}=0.11)$. No opinions were requested to OHNs in 
Table 1. OH activities of OHPs and OHNs in companies employing a full-time OHP and those employing a part-time OHP

\begin{tabular}{|c|c|c|c|c|c|}
\hline \multirow{2}{*}{ Items of $\mathrm{OH}$ duties } & \multicolumn{2}{|c|}{ Full-time-OHP company $(n=6)$} & \multicolumn{2}{|c|}{ Part-time-OHP company $(n=12)$} & \multirow{2}{*}{$P$ value } \\
\hline & Number & $(\%)$ & Number & $(\%)$ & \\
\hline \multicolumn{6}{|l|}{$\mathrm{HCM}$} \\
\hline Work & 6 & 100 & 12 & 100 & \\
\hline Attend by OHP more than half & 5 & 83 & 5 & 42 & 0.24 \\
\hline \multicolumn{6}{|l|}{ OHN activity in HCs } \\
\hline Attend meetings & 2 & 33 & 10 & 83 & 0.11 \\
\hline Assignment roles in $\mathrm{HC}$ & 1 & 17 & 7 & 58 & 0.24 \\
\hline Opportunity to make speeches, reports, presentations & 1 & 17 & 8 & 67 & 0.13 \\
\hline Opinions requested & 0 & 0 & 6 & 50 & 0.11 \\
\hline Propose $\mathrm{OH}$ services & 1 & 17 & 4 & 33 & 0.85 \\
\hline Reports sent to OHN & 4 & 67 & 11 & 92 & 0.50 \\
\hline Office tasks & 1 & 17 & 5 & 42 & 0.60 \\
\hline Introduction of new $\mathrm{OH}$ information & 1 & 17 & 3 & 25 & 0.84 \\
\hline Explanation of health examination & 1 & 17 & 6 & 50 & 0.39 \\
\hline Health education & 0 & 0 & 3 & 25 & 0.50 \\
\hline Conduct meetings & 0 & 0 & 2 & 17 & 0.79 \\
\hline \multicolumn{6}{|l|}{ Walk through surveys } \\
\hline Made by OHP more than once a month & 5 & 83 & 5 & 42 & 0.24 \\
\hline Made by OHN & 3 & 50 & 8 & 67 & 0.86 \\
\hline
\end{tabular}

OH: Occupational Health; OHP: Occupational Health Physician; OHN: Occupational Health Nurse; HC: Health Committee; HCM: Health Committee Meeting.

companies employing full-time OHPs, but $50 \%$ of OHNs were requested their opinions in those employing part-time OHPs ( $\mathrm{p}=0.11$ ). The HC, which is one of the most important intra-industrial $\mathrm{OH}$ activities, may demand a specialized position for OHNs apart from OHPs, particularly in companies employing only part-time OHPs.

Although the number of companies included in this survey was small, the results indicate a potentially important role for OHNs in the $\mathrm{HC}$ because there were so few reports on present conditions of OHN activity related to HCs in Japan. A previous study in Japan showed that half of the OHNs working in companies with less than 1,000 employees were not involved in the $\mathrm{HC}$ and that $40 \%$ of subjects felt dissatisfaction with their company policy toward $\mathrm{OHN}$ attendance in the $\mathrm{HCM}^{3}$. This result indicates a gap in thinking between OHNs and company managers about necessary $\mathrm{OH}$ services. We suggest that current conditions in $\mathrm{OH}$ be frequently reported to promote active discussion among OH-related staff including OHNs and company managers. An employer is required to hold an HCM at least once a month, and the occupational safety and health law determines which individuals will attend. For example, half of the attendants are to be selected from the union or whatever organization serves as the company union. But there is no law at present that mandates the OHN's attendance at the
HCM. Moreover, a local OH survey shows only half of part-time OHPs attended to the HCM, even though OHPs are demanded by the law to attend ${ }^{5}$. The similar $\mathrm{OH}$ condition of the OHPs' attendance to the HCM is not rare nationwide in Japan, particularly true in companies employing part-time OHPs only. Under the current $\mathrm{OH}$ conditions, OHNs with professional knowledge and skills may make up for the $\mathrm{OH}$-relating issues as a member of the HC. We suggest that an OHN attendance at the HCM, the most influential $\mathrm{OH}$ consultation organ, is important for employers and their workers to develop better $\mathrm{OH}$ services within the company particularly when a part-time OHP only is employed.

Whereas periodic walk through surveys by an OHP are prescribed by the law, OHNs are not required to make similar visits. However, $50.0 \%$ of OHNs in companies employing full-time OHPs and $66.7 \%$ of OHNs in companies with parttime OHPs conduct walk through surveys. Even though OHPs are obligated to conduct walk through surveys in their companies, more than half of the part-time OHPs did not do so at the required frequency. There might be a practical reason that OHPs do not have enough time, however, they are demanded to walk through worksite periodically once a month even no reason exists. In our survey, this tendency was found more often in companies employing part-time 
OHPs than full-time OHPs. Then we consider that OHNs may support part-time OHP's worksite visits in certain circumstances. Of course, it is necessary for OHNs to know worksite conditions to provide $\mathrm{OH}$ management, regardless of OHP performance. We suggest that OHNs, especially in those companies employing part-time OHPs, acquire expertise in conducting walk through surveys.

Under the present weakened economic situation, it is anticipated that large Japanese companies will scale down in the number of employees and that smaller related companies will be created as a result of restructuring. According to Japanese occupational safety and health law, a company having more than 1,000 employees is required to employ a full-time OHP to manage $\mathrm{OH}$ services. In companies with less than 1,000, but more than 50 employees, the employer is required to appoint an OHP but not necessarily on full-time basis. However, there is no regulation designating how many days per month a part-time OHP is required to work at the company. Indeed, the frequency of company work for part-time OHPs varied from $2 \mathrm{~d}$ per $\mathrm{yr}$ to $2 \mathrm{~d}$ per wk, according to our survey. Under the present $\mathrm{OH}$ conditions, we suggest that OHNs in Japan become even more involved as key professionals in $\mathrm{OH}$ fields because they work regularly in their companies and are therefore familiar with employees' affairs.

Karoshi or "death from overwork", has added a significant problem to the recession in Japan, one that requires an urgent solution $^{6}$. Karoshi problem, which includes cardiovascular disease and self-destruction ${ }^{7}$, has increased as the recession has worsened. The number of events, including cardiovascular disease and self-destruction, applied for labor accident compensation, and the number of recognition from labor standards inspection office were 508 and 94 in 1998, 648 and 95 in 1999, 829 and 121 in 2000, and 945 and 213 in 2001, respectively ${ }^{8}$. To prevent karoshi, employees' affairs related to working conditions should be monitored in detail. OHPs take a leading role in various countermeasures against karoshi because they know worksite condition, working condition, and workers' health condition. However, our present survey have shown that less than half of part-time OHPs attend HCs and perform walk through survey, supposing that enough countermeasures are not offered. Then, particularly in companies with part-time OHPs, there are cases where the OHNs have to give some advice about health maintenance in various situations because they might know about worksite condition of the company, employees' working conditions, their lifestyle, and sometime about their families. Under the present conditions, we consider that the OHNs should pursue components of $\mathrm{OH}$ specialization for better performance.

Therefore, OHNs in the future will need an environment that supports education to improve their skills in $\mathrm{OH}$ communication techniques, such as making presentations and introducing new practices. Inter-personal evaluation and decision-making skills are also to be included to improve professional abilities ${ }^{9}$. The need for change in the OHN's work role and education has been pointed out in Finland ${ }^{10)}$. In this study, the number of companies in a local area surveyed was small. However, our present results are not different from a tendency observed in the present Japanese society. Japan will also need OHNs to acquire greater skills as the society sinks into recession.

In conclusion, our survey results show that OHNs working in companies with part-time OHPs were more active in the HCs than OHNs working in companies with full-time OHPs. In the future, OHNs, particularly those working with a parttime OHP, will have an important role in the $\mathrm{HC}$ and developing $\mathrm{OH}$ services for their companies. Therefore, OHNs are advised to actively pursue an education containing components of $\mathrm{OH}$ specialization for better performance in $\mathrm{OH}$ services. More precise research will be needed account large number of companies to address in-depth the role of OHNs in $\mathrm{OH}$ services.

\section{References}

1) Nagata $S$, Ishibashi $S$ (2001) Mental health and stress. J Jpn Med Assoc 126, 359-63 (in Japanese).

2) Takahashi $K$ (2000) Past, present and future trends of occupational health in Japan, as at 1998. Occup Med 50, 437-9.

3) Isono $F$ (1997) Job conditions and the current conception of occupational health nurses in industries having fewer than 1,000 employees. San Ei Shi 39, 203-8 (in Japanese with English abstract).

4) Ikushima M, Ishihara I, Kawamoto R (2002) A survey of public health nurses regarding their awareness of the abilities, skill and knowledge necessary to fulfill an occupational health nurse's role, and proposal of a new curriculum for occupational nurse specialists at the master level. J UOEH 24, 313-25 (in Japanese with English abstract).

5) Takeda N, Kawada K, Emi G, Ujike M, Kageyama H (1999) Present status and problems of occupational health doctors. J District Environ/ Health/ Welfare Research 3, 132-5 (in Japanese).

6) Nishiyama K, Johnson JV (1997) Karoshi-death from overwork: occupational health consequences of 
Japanese production management. Int J Health Serv 27, 625-41.

7) Uehata $T$ (2001) Karoshi and work-culture in Japan. Education and Medicine 49, 737-45 (in Japanese).

8) Uehata $T$ (2002) A revision of labor compensation standards of karoshi and preventive measures. J Public Health Practice 66, 837-42 (in Japanese).
9) Naumanen-Tuomela P (2001) Occupational health nurses' work and expertise in Finland: occupational health nurses' perspective. Public Health Nurs 18, 10815.

10) Rossi K, Heinonen K, Heikkinen MR (2000) Factors affecting the work of an occupational health nurse. Occup Med 50, 369-72. 